\title{
Optical Properties of Carbon Nanotubes in a Composite Material : the Role of Dielectric Screening and Thermal Expansion.
}

\author{
S. Berger ${ }^{1}$, F. Iglesias ${ }^{1}$, P. Bonnet ${ }^{1}$, C. Voisin ${ }^{1}$, G. Cassabois ${ }^{1}$, J-S. Lauret ${ }^{2}$, C. Delalande ${ }^{1}$, and P. Roussignol ${ }^{1}$ \\ 1 Laboratoire Pierre Aigrain, École Normale Supérieure, \\ CNRS UMR8551, Université Pierre et Marie Curie, \\ Université Paris Diderot, 24 rue Lhomond, Paris, France and \\ 2 Laboratoire de Photonique Quantique et Moléculaire, CNRS UMR8537, \\ Institut d'Alembert, Ecole Normale Supérieure de Cachan, \\ 61 avenue du Président Wilson, Cachan, France
}

\begin{abstract}
We report on environmental effects on the optical properties of single-wall carbon nanotubes in a gelatin-based composite material designed to foster their photoluminescence. We show that the dielectric screening of excitons due to the surrounding medium is responsible for a sizeable shift of the luminescence lines which hardly depends on the tube geometry. In contrast, the temperature dependence (from 4 to $300 \mathrm{~K}$ ) of the luminescence is clearly chirality dependent : the first and second excitonic lines shift in opposite directions with a magnitude that can be related quantitatively to a strain-induced modification of the electronic structure due to an expansivity mismatch between the nanotube and the matrix.
\end{abstract}

PACS numbers:

\section{INTRODUCTION}

Single-Wall Carbon Nanotubes (SWCNTs) which consist of a single enrolled graphene sheet are natural prototypes of quasi-unidimensional objects and therefore have drawn considerable attention in fields ranging from very fundamental physics to industrial applications. As for optical properties SWCNTs have demonstrated unique features such as unprecedented exciton binding energies [1, 2], large nonlinear susceptibilities [3] and emission/absorption spectra that allow the assignment of each optical feature to a specific chirality [4]. SWCNTs have also been successfully integrated in electro-optical devices such as field-effect transistors for light emission or detection [5]. However, since they are intrinsically made of surface atoms, SWCNTs strongly and inevitably interact with their environment. A dramatic illustration of this singular property is the total quenching of luminescence that occurs when nanotubes aggregate into bundles [6] or lie on a substrate [7]. This has hampered the development of optical studies of nanotubes until an efficient way of producing isolated nanotubes had been proposed [8]. The most popular of these methods for bulk samples is based on a chemico-physical insulation of nanotubes encased in micelles of surfactant with possible further processing and integration in various matrices such as polymers or gelatins $[9,10]$. These composite materials are of technological interest since they are much easier to handle than solutions and allow to vary external parameters such as temperature, strain, electrical gating...

In this paper, we study the photoluminescence properties of SWCNTs in a composite material optimized for optical purposes. We show that even for such isolated nanotubes the environment plays a key role in their optical properties. The zero order effect is a sizeable shift of the resonances due to environment-induced dielectric screening of the Coulomb interactions together with a broadening of the lines. When cooling the sample down to cryogenic temperatures, we show that the expected intrinsic change of the electronic structure is completely masked by subtle environment-induced effects : the thermal expansivity mismatch between the nanotube and the surrounding matrix induces considerable stress which in turn modifies the electronic structure of the tube in a way that is remarkably accounted for by calculations of uniaxial strain-induced changes of the band-structure $[11,12]$.

\section{SAMPLES}

The samples consist of a gelatin-based composite material doped with HiPCO SWCNTs (purchased from Carbon Nanotechnologies Inc.). Isolated nanotubes encased in micelles of surfactant are obtained following standard methods based on ultrasonication in an aqueous solution of Sodium Dodecyl Sulfate (SDS) (1 wt \%)[8]. After ultracentrifugation at $200000 \mathrm{~g}$ for $4 \mathrm{~h}$, the supernatant is collected. As pointed by several studies, this method gives suspensions strongly enriched in isolated single nanotubes, but the suspension still contains a non negligible amount of small bundles [10, 13]. For similar samples we had estimated the relative amount of single tubes to be of the order of $10 \%$ [10]. We further incorporate commercial dehydrated gelatin in the solution at $70^{\circ} \mathrm{C}(10 \mathrm{mg} / \mathrm{ml})$. A little amount of this gelatin enriched solution is dropped on a quartz substrate and dried at room temperature.

\section{RESULTS AND DISCUSSION}

\section{A. Photoluminescence Properties}

Fig. 1 shows that gelatin-based samples are an excellent choice for optical studies of carbon nanotubes : the 
photoluminescence (PL) efficiency of such a composite material remains comparable to the one of the initial suspension over time whereas a simple deposition and drying of a drop of the initial suspension leads to a drastic quenching of the PL (more than one order of magnitude) when the solvent is evaporated. This is most likely due to a collapse of the micelles at high concentration which leads to reaggregation of the tubes into bundles. In contrast the gelatin network is known to remain highly hydrated [14] and therefore preserves the micelle structure and the insulation of the nanotubes. Adding some gelatin into a suspension of isolated nanotubes turns out to be one of the most efficient methods published so far to obtain a solid-state composite preserving the luminescence properties of nanotubes. Furthermore, these samples can endure several cryogenic cooling cycles without any deterioration of their optical properties.

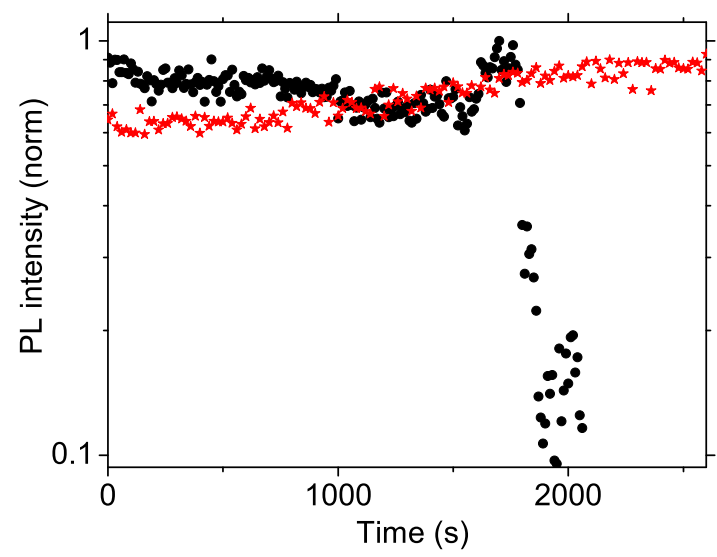

FIG. 1: Temporal evolution of the photoluminescence intensity (logarithmic scale) after deposition on a glass substrate of a drop of a suspension of SWCNTs in water (black dots) and a drop of gelatin-enriched material (red stars) at room temperature. The luminescence intensity of the gelatin-based sample remains constant over time whereas the luminescence of the suspension is reduced by one order of magnitude when the solvent is evaporated. The transient increase of the signal is likely due to a lens effect in the drying drop changing the collection efficiency of the setup.

Fig. 2 shows a typical PL excitation (PLE) map of a gelatin-based sample doped with HiPCO nanotubes. Each bright spot corresponds to the luminescence from the so-called $\mathrm{S}_{11}$ excitonic level resonantly excited on the $\mathrm{S}_{22}$ excitonic level of a given semi-conducting nanotube family (i.e. defined by a pair of chiral indices $(n, m))$. Following the assignment scheme proposed by Bachilo et al. [4], we assigne each spot to the chiral indices reported on the map. Additional chiral families are accessible in the same detection range by tuning the excitation energy to lower values (around $1.5 \mathrm{eV}$, not shown in Fig. 2).

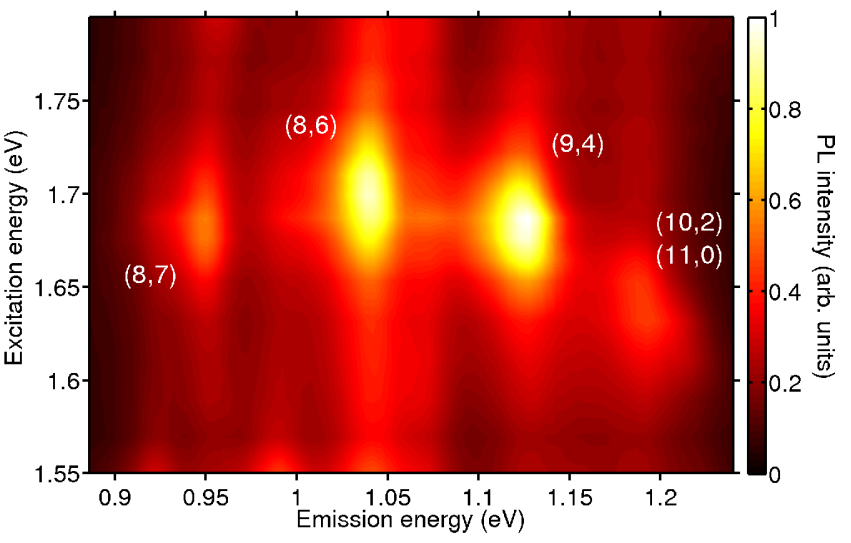

FIG. 2: Photoluminescence intensity at $10 \mathrm{~K}$ of isolated HiPCO SWCNTs embedded in a gelatin matrix plotted as a function of emission and excitation energies. By comparison to the semi-empirical Kataura plot [4], the PL spots are assigned to a given SWCNT chirality.

\section{B. Dielectric Screening}

A comparison of the PL spectra of suspensions with those of gelatin-based samples brings a striking evidence of the high sensitivity of nanotubes to their environment. The data displayed in Fig. 3 show a global red shift of the PL lines of the nanotubes embedded in gelatin as compared to the ones of the nanotubes in aqueous suspension. This corresponds to a red shift of the order of $15 \pm 4 \mathrm{meV}$ of the $\mathrm{S}_{11}$ exciton level. Data on the $\mathrm{S}_{22}$ levels were obtained by means of PLE measurements on the same species and reveal a red-shift of the same magnitude. Similar effects have been reported upon changing the solvant of NT suspensions $[15,16]$. We interpret this effect as a consequence of the dielectric screening of the Coulomb interaction in the nanotube through its environment due to the spill-out of the wave-function of the exciton. Two main effects drive the exciton energy i.e. the direct Coulomb interaction and the self-energy. The former tends to lower the exciton energy (binding energy) whereas the latter leads to an enhancement of the bandgap. Theoretical and experimental studies have shown that the latter dominates for SWCNTs [17-19]. Therefore an increase of the dielectric constant results in a red shift of the line.

Perebeinos et al. have proposed a scaling law for the binding energy of the exciton $\left(E_{b} \propto \epsilon^{-\alpha}\right.$ with $\left.\alpha=1.4\right)$ where $\epsilon$ is an effective dielectric constant [20]. In the case of small diameter nanotubes such as the present ones $\left(d_{t} \sim 0.9 \mathrm{~nm}\right)$, the main contribution to the dielectric constant is the one of the environment [20]. Lefebvre et al. have proposed to generalize this approach for the self-energy $\left(E_{s} \propto \epsilon^{-\beta}\right.$ with $\left.\beta=1\right)$ [21]. Therefore the change of the exciton energy upon a relative change of the dielectric constant reads : $\Delta E=\frac{\Delta \epsilon}{\epsilon}\left(\alpha E_{b}-\beta E_{s}\right)$. 
The binding energy for nanotubes in micelles has been previously measured by two-photon PLE measurements and is of the order of $380 \mathrm{meV}$ for $0.9 \mathrm{~nm}$ diameter nanotubes $[1,2]$. The self-energy correction has been estimated by fine spectroscopy analysis of a temperatureinduced bandgap shift transition and turns out to be of the order of $E_{s} \sim 650 \mathrm{meV}$ in solution [21]. From the experimental line-shift observed in Fig. 3, we deduce that the relative change in the dielectric environment (gelatin vs suspension) of the tube is of the order of $+15 \%$.

Let us examine the possible origin of this modification of the dielectric screening. The dielectric constant of interest in this problem is the one probed by the exciton wave-function, in the very local environment of the tube. In view of the large exciton binding energy in carbon nanotubes, one has to consider an optical dielectric constant rather than a static one [22]. This quantity is difficult to estimate in a complex environment involving long-chain-molecules in a disordered medium. However we can estimate upper and lower bounds in each environment. Molecular dynamics simulations by O'Connel et al. [8] show that for nanotubes in a micelle the local environment of the tube within a distance of $1 \mathrm{~nm}$ (typical exciton size [1]) is made of the close-packed alkyl chains of SDS molecules with a carbon density of the order of $30 \mathrm{~nm}^{-2}$. The corresponding dielectric constant is of the order of 1.8 [23]. Note that due to the sharp density profile, the value is presumably significantly dependent on the molecular arrangement. In the gelatin environment the molecular pressure from the matrix can induce a densification of the micelle and consequently an increase of the local dielectric constant. The molecular dynamics simulations in [8] show that a moderate rearrangement of the micelle structure can easily lead to a $10 \%$ increase in the local density. The extreme case would correspond to the dissociation of the micelles and direct enclosure of the nanotubes in the hydrated gelatin matrix. The dielectric constant of the latter is of the order of 2.3 (refractometric measurements [24]). Compared to the suspension environment this corresponds to a relative increase of the dielectric constant of the order of $25-30 \%$ in qualitative agreement with the spectroscopic estimate. From this analysis we can deduce a quantity of practical interest : the sensitivity of the exciton energy upon a change of surrounding dielectric constant $:\left.\frac{d S_{11}}{d \epsilon}\right|_{\epsilon=2} \sim 45 \mathrm{meV}$ (obtained for a typical dielectric constant of 2 ).

\section{Line Broadening}

An other consequence of the insertion of nanotubes in a gelatin matrix is the broadening of the emission lines (cf. Fig. 3). The effect is especially clear for the $(10,2)$ and $(9,4)$ lines, although the former is partially quenched by off resonance excitation conditions due to the shift of $\mathrm{S}_{22}$ in gelatin. In average the line broadening is of the order of 5 to $10 \mathrm{meV}$ that is up to $50 \%$ of

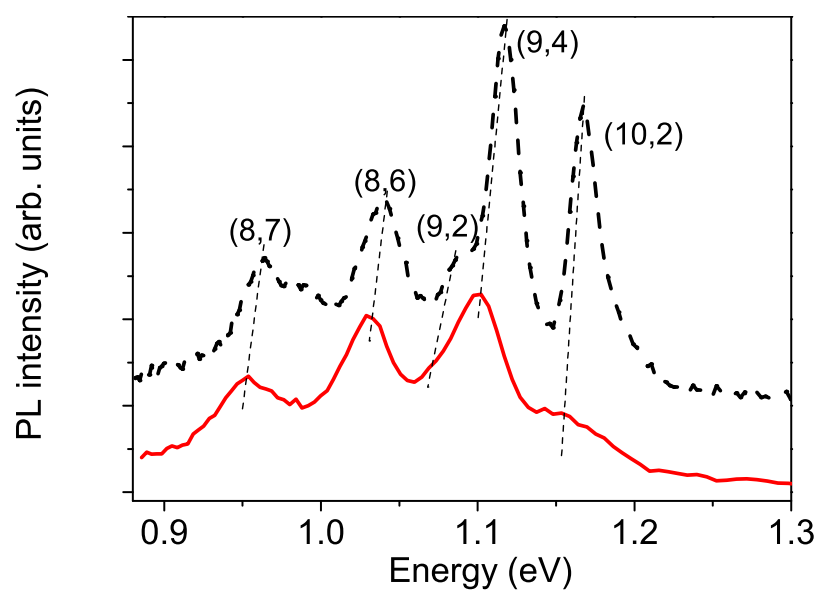

FIG. 3: Photoluminescence spectrum of isolated HiPCO SWCNTs in an aqueous suspension (black dashed line) or embedded in a gelatin matrix (red solid line) excited at $1.69 \mathrm{eV}$ at room temperature. The data show a global red shift of about $15 \mathrm{meV}$ for gelatin-embedded nanotubes.

the initial linewidth. As for $\mathrm{S}_{22}$ transitions, our measurement is hampered by the large background and an initial linewidth of the order $100 \mathrm{meV}$. We see no significant extra-broadening within a $10 \mathrm{meV}$ experimental resolution. The broadening of $\mathrm{S}_{11}$ transitions may be related to several mechanisms. Bearing in mind that the PL lines stem from an ensemble of nanotubes, the extrabroadening can be of inhomogeneous nature : the global red shift mentioned previously corresponds to an increase of the average dielectric function of the environment of the tube but local variations can lead individual lines to deviate from the mean position and result in an extrainhomogeneous broadening. This would explain why the global red-shift and the extra-broadening are of the same order of magnitude. Another type of broadening mechanism especially relevant for nanostructures is related to environment-induced dephasing processes. These latter may involve a more efficient coupling to the phonon modes of the solid matrix (with respect to a liquid surrounding) or the presence of fluctuating photo-induced charges in the local environment of the nanotube. This latter effect is well established for nanostructures embedded in matrices [25] and has been previously evoked to account for spectral diffusion and blinking phenomema at the single nanotube level [26].

\section{Thermal Expansion}

In contrast to suspensions, solid state samples such as nanotube-doped gelatin samples allow low temperature measurements. As reported elsewhere, in the very low temperature range ( 4 to $40 \mathrm{~K}$ ), these studies have permitted to gain valuable information about the exciton structure and the existence of a low-lying dark state [10]. 
In this study, we focus on the intermediate temperature range and the shift of the $\mathrm{S}_{11}$ and $\mathrm{S}_{22}$ lines. Fig. 4 shows a set of PL spectra for temperatures ranging from 10 to $300 \mathrm{~K}$. A clear spectral shift of the lines (associated to a specific chiral family) is observed. However, in contrast to the previous graph (Fig. 3), this is not a global shift of the whole spectrum but a highly chiral dependent shift : the amplitude of the shift strongly varies from one line to another and even the sign of the shift changes. Table I gives a summary of the line-shifts observed when cooling the sample down to $150 \mathrm{~K}$, for both the $\mathrm{S}_{11}$ and $\mathrm{S}_{22}$ (when observable) transitions. Although a limited range of chiral families is available, some clear trends show up : first $\mathrm{S}_{11}$ and $\mathrm{S}_{22}$ systematically shift in opposite directions. of the tubes : nanotubes with $q=n-m=1(\bmod 3)$ show a positive shift for $S_{11}$ (and negative for $S_{22}$ ) whereas tubes with $q=n-m=-1(\bmod 3)$ show a negative shift for $\mathrm{S}_{11}$ (and positive for $\mathrm{S}_{22}$ ). Finally, the magnitude of the spectral shift clearly depends on the chiral angle and is larger for smaller chiral angles (close to zig-zag geometry). The magnitude of the spectral shift of the $\mathrm{S}_{11}$ line as a function of the chiral angle is displayed in Fig. 5. It turns out to be proportional to $\cos 3 \theta$. Similar effects have been previously reported for polymer wrapped nanotubes or frozen suspensions of nanotubes [9, 27, 30]. Second, the sign of the shift is related to the family type

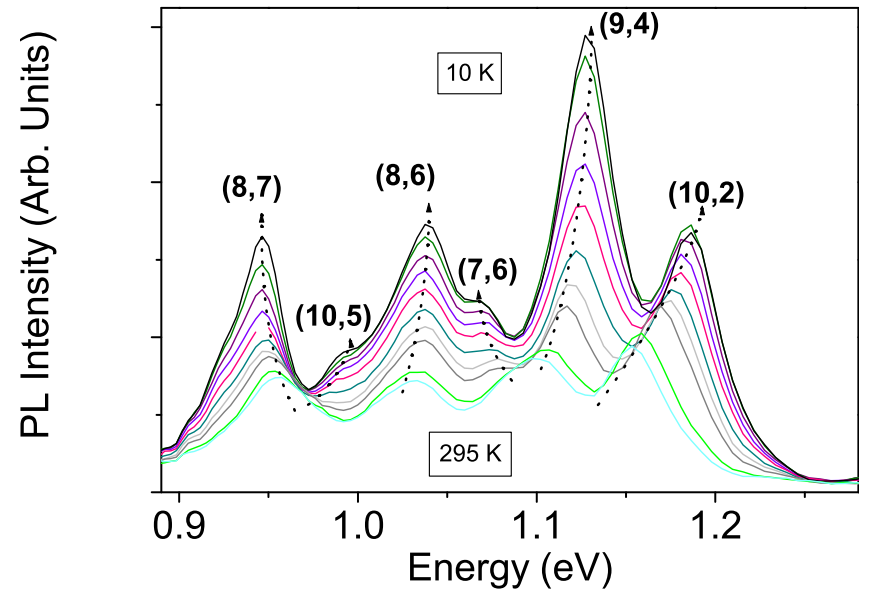

FIG. 4: Photoluminescence spectrum of isolated HiPCO SWCNTs embedded in a gelatin matrix as a function of temperature. The excitation energy is $1.7 \mathrm{eV}$. The lower the temperature, the larger the PL intensity.

be understood as a consequence of a moderate uniaxial strain $(\sigma)$ applied on the tubes at low temperatures.

Due to the large aspect ratio of the tube and the large Young modulus mismatch between the matrix and the tube, the thermally induced deformation of the tube is

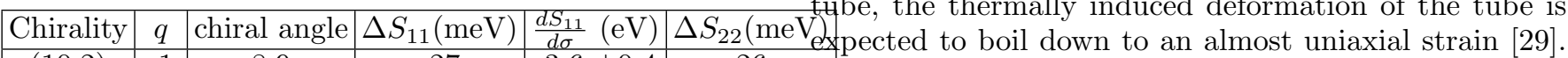

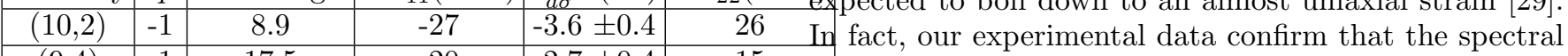
\begin{tabular}{c|c|c|c|c|c|}
\hline$(9,4)$ & -1 & 17.5 & -20 & $-2.7 \pm 0.4$ & 15 \\
\hline
\end{tabular}

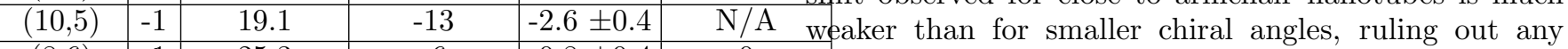
\begin{tabular}{c|c|c|c|c|cc}
$(8,6)$ & -1 & 25.3 & -6 & $-0.8 \pm 0.4$ & 0 & significant torsional or radial strain contribution. This
\end{tabular}

\begin{tabular}{|c|c|c|c|c|c|}
\hline$(7,6)$ & +1 & 27 & 5 & $0.7 \pm 0.4$ & effect is related to a thermal expansion coefficient mis- \\
\hline
\end{tabular}

\begin{tabular}{l|l|l|l|l|l|l}
$(8,7)$ & +1 & 27.8 & 6 & $0.9 \pm 0.4$ & -6 \\
\hline
\end{tabular}

TABLE I: Spectral shifts of the $\mathrm{S}_{11}$ line for several nanotubes under thermally induced strain. $q$ stands for $n-m(\bmod 3)$. $\Delta S_{11}$ stands for $\mathrm{S}_{11}(300 \mathrm{~K})-\mathrm{S}_{11}(150 \mathrm{~K})$. A negative strain is induced at low temperature.

L. Yang et al. [11, 12] have studied theoretically the effect of uniaxial and torsional strain on the band gap of SWCNTs. This study has been recently adjusted by Huang et al.[28] to account for the internal sublattice relaxation of the graphitic structure in the case of uniaxial strain $(\sigma)$ and leads to :

$$
\frac{d S_{i i}}{d \sigma}=0.57 q(-1)^{i}(1+\nu) 3 t_{0} \cos (3 \theta)
$$

where $\nu$ is the Poisson ratio and $t_{0}$ the electron hopping parameter. This study turns out to be an excellent framework to interpret our experimental results. Especially, the sign of the spectral shifts reported here (including its dependence on the tube family $(q)$ and the transition number $i\left(\mathrm{~S}_{11}\right.$ vs $\left.\mathrm{S}_{22}\right)$ ) as well as the magnitude of the effect as a function of the chiral angle $(\theta)$ can match between the nanotube and the surrounding matrix $[30,31]$. The linear thermal expansivity is of the order of $\alpha_{t}=-5.10^{-6} \mathrm{~K}^{-1}$ for nanotubes [32] whereas for water ice it is slightly negative below $60 \mathrm{~K}$ and varies at $200 \mathrm{~K}$ [33]. Interestingly the observed spectral shift versus temperature (cf. Fig. 6) shows a plateau between 4 and $50 \mathrm{~K}$, which corresponds to the temperature range where the thermal expansivities of water and nanotubes almost balance each other. Conversely the thermallyinduced spectral shift increases with temperature above $50 \mathrm{~K}$ as expected from the increasing value of the matrix expansivity. For further quantitative analysis we focus on the range $150-300 \mathrm{~K}$ where we fit the thermally-induced spectral shift to a linear law. The strain-induced spectral shift is deduced from that fit assuming a full load transfer from the matrix and an average value of the matrix expansivity of $4.5 \times 10^{-5} \mathrm{~K}^{-1}$ [33].

We obtain : $\frac{d S_{11}}{d \sigma}=\frac{d S_{11}}{\Delta \alpha d T} \sim-3.6 \mathrm{eV}$ for the $(10,2)$ nanotube and $-0.8 \mathrm{eV}$ for the $(8,6)$ (cf. Table I). We estimate that the strain applied to the tube when the sample is cooled down to $150 \mathrm{~K}$ is of the order of $-7.510^{-3}$, which corresponds to an applied pressure of $7.5 \mathrm{GPa}$ if one assumes a Young modulus of $1 \mathrm{TPa}$ for nanotubes [34]. Although the absence of jumps or plateaux in the almost linearly from 0 at $60 \mathrm{~K}$ to $\alpha_{w}=+4.10^{-5} \mathrm{~K}^{-1}$ 


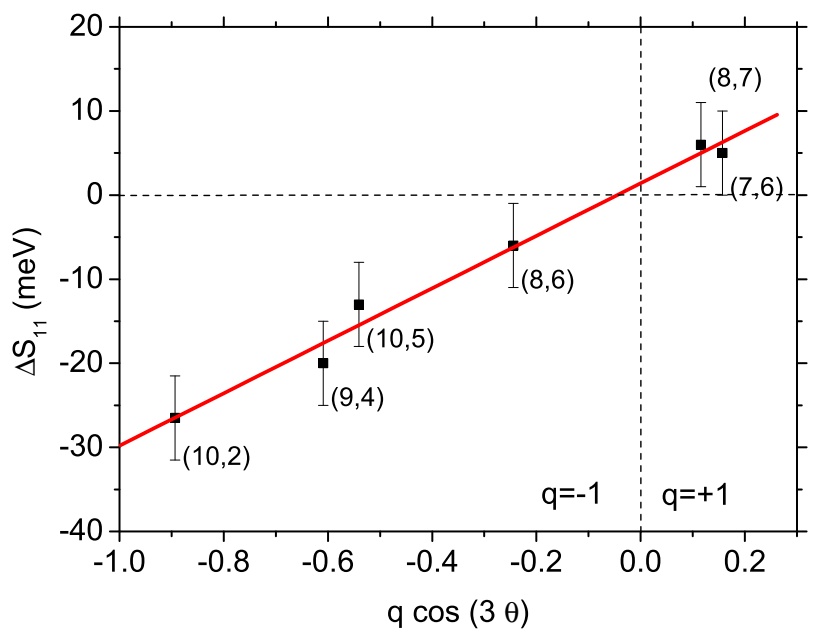

FIG. 5: Energy shift of the emission line of several nanotubes when the sample is heated from $150 \mathrm{~K}$ through $300 \mathrm{~K}$ plotted against the geometric parameter $q \cos (3 \theta)$. This temperature change corresponds to a positive strain of the order of $0.7 \%$.

range 150-300 K supports the assumption that the load transfer is high, one cannot exclude that little slips of the nanotubes relative to the matrix occur at the single tube level, which are smoothed out by the ensemble measurement. Therefore the strain applied to the tube is likely to be overestimated and conversely for the strain-induced spectral shift.

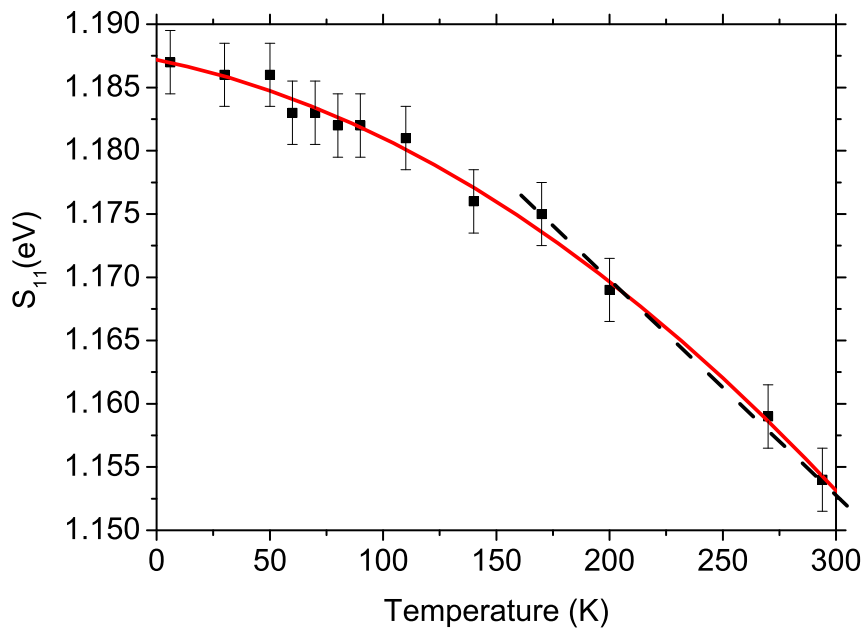

FIG. 6: Emission energy $\left(\mathrm{S}_{11}\right)$ of $(10,2)$ nanotubes as a function of temperature. The excitation energy is $1.7 \mathrm{eV}$. The full red line is a parabolic fit whereas the black dashed line is a linear fit for the range $[150-300 \mathrm{~K}]$ used to estimate $\frac{d S_{11}}{d \sigma}$ (see text).
These results can be compared with recently published data obtained in experimental conditions where the strain is applied in a different manner : either through the deformation of a polymer matrix host [35] or through direct mechanical stretching of the nanotube [28]. Since all those publications agree on the linearity of $\frac{d S_{11}}{d \sigma}$ with the geometrical factor $q \cos (3 \theta)$, we can compare the magnitude of the effect by measuring the slope (cf. Fig. 5). The slope reported by Leeuw et al. [35] is of the order of $9 \mathrm{eV}$, whereas the one reported by Huang et al.[28] is of the order of $4.5 \mathrm{eV}$. Our own value is $4.2 \mathrm{eV} \pm 0.5$. According to Eq.(1) and using a commonly accepted value of 0.15 for $\nu$ [34] we deduce an estimate of the C-C hopping integral of $2.2 \pm 0.3 \mathrm{eV}$. As discussed above, due to possible imperfect load transfer this value is likely to be underestimated ; however it is still in good agreement with usual values found in the literature (ranging from 2.4 to $3 \mathrm{eV}$ ) [36, 37].

We can further note that the absence of chiral dependent spectral shift for gelatin samples (as compared to suspensions) at room temperature contrasts with what is reported for other polymers [9]. This means that no sizeable stress is applied to the tube from the matrix at room temperature, which is consistent with the picture of a highly hydrated material in which the micelle structure is preserved.

\section{CONCLUSION}

To summarize, we have investigated the optical properties of carbon nanotubes embedded in a functional composite material. This study brings evidence for the extreme sensitivity of nanotubes to their environment. In particular, we have shown that any possible intrinsic effect is overwhelmed by extrinsic effects such as excitonic dielectric screening and strain induced spectral shifts. The former leads to a red shift of the excitonic lines upon an increase of the local dielectric constant. The strain induced spectral shifts and their chiral dependence reveal important information about the mechanical properties of such composite material and their relationship with the band structure of the nanotubes. Furthermore it provides evidence for an efficient load transfer from the matrix to the tube, which is of outmost importance for mechanical reinforcement applications.

\section{ACKNOWLEDGEMENTS}

This work has been done in the framework of the GDRE $\mathrm{n}^{\circ} 2756$ 'Science and applications of the nanotubes - NANO-E'.
[1] F. Wang, G. Dukovic, L.E. Brus, T.F. Heinz, Science 308, $838(2005)$
[2] J. Maultzsch, R. Pomraenke S. Reich, E. Chang, D. 
Prezzi, A. Ruini, E. Molinari, M. Strano, C. Thomsen and C. Lienau, Phys. Rev. B 72, 241402 (2005)

[3] J-S. Lauret, C. Voisin, G. Cassabois, J. Tignon, C. Delalande, Ph. Roussignol, O. Jost and L. Capes, Appl. Phys. Lett. 85, 3572, (2004)

[4] S.M. Bachilo, M.S. Strano, C. Kittrell, R.H. Hauge, R.E. Smalley, R.B. Weisman, Science 298, 2361 (2002)

[5] J.A. Misewich, R. Martel, Ph. Avouris, J. C. Tsang, S. Heinze and J. Tersoff, Science 300, 783 (2003)

[6] J-S. Lauret, C. Voisin, G. Cassabois, C. Delalande, P. Roussignol, O. Jost and L. Capes, Phys. Rev. Lett. 90, 057404 (2003)

[7] J. Lefebvre, Y. Homma and P. Finnie, Phys. Rev. Lett. 90, 217401 (2003)

[8] M.J. O'Connell, S.M. Bachilo, C.B. Huffman, V.C. Moore, M.S. Strano, E.H. Haroz, K.L. Rialon, P.J. Boul, W.H. Noon, C. Kittrell, J.P. Ma, R.H. Hauge, R.B. Weisman, R.E. Smalley, Science 297, 593 (2002)

[9] L-J. Li, R.J. Nicholas, R.S. Deacon and P. A. Shields, Phys. Rev. Lett. 93, 156104 (2004)

[10] S. Berger, C. Voisin, G. Cassabois, C. Delalande, P. Roussignol and X. Marie, Nano. Lett. 7, 398 (2007)

[11] L. Yang, M.P. Anantram, J. Han and J.P. Lu, Phys. Rev. B 60, 13874 (1999)

[12] L. Yang and J. Han, Phys. Rev. Lett. 85, 154 (2000)

[13] P.H. Tan, A.G. Rozhin, T. Hasan, P. Hu, V. Scardaci, W.I. Milne and A.C. Ferrari, Phys. Rev. Lett. 99, 137402 (2007)

[14] P. V. Kozlov and G. I. Burdygina, Polymer 24, 651 (1983)

[15] O. Kiowski, S. Lebedkin, F. Hennrich, Sharali Malik, H. Rösner, K. Arnold, C. Sürgers and M.M. Kappes, Phys. Rev. B 75, 075421 (2007)

[16] Y. Ohno, S. Iwasaki, Y. Murakami, S. Kishimoto, S. Maruyama and T. Mizutani, Phys. Stat. Sol. b 244, 4002 (2007)

[17] F. Wang, M.Y. Sfeir, L. Huang, X.M.H. Huang, Y. Wu, J. Kim, J. Hones, S. O'Brien, L.E. Brus and T.F. Heinz, Phys. Rev. Lett. 96, 167401 (2006)

[18] C.D. Spataru, S.b Ismail-Beigi, L.X. Benedict, and S.G. Louie, Phys. Rev. Lett. 92, 077402 (2004)

[19] T. Ando, J. Phys. Soc. Jap. 66, 1066 (1997)
[20] V. Perebeinos, J. Tersoff and P. Avouris, Phys.Rev. Lett. 92, 257402 (2004)

[21] J. Lefebvre and P. Finnie, Nano Lett. 8, 1890 (2008)

[22] C.F. Klingshirn in Semiconductor optics, SpringerVerlag, Berlin Heidelberg (1995)

[23] T. M. Aminabhavi, V. B. Patil, M. I. Aralaguppi, and H. T. S. Phayde, J. Chem. Eng. Data 41, 521 (1996)

[24] K. Nikolova, I. Panchev and S. Sainov, J. Optoelectronics and Adv. Mat. 7, 1439 (2005)

[25] A. Berthelot, I. Favero, G. Cassabois, C. Voisin, C. Delalande, P. Roussignol, R. Ferreira, J.-M. Gérard, Nature Physics 2, 759 (2006)

[26] K. Matsuda, Y. Kanemitsu, K. Irie, T. Saiki, T. Someya, Y. Miyauchi and S. Maruyama, Appl. Phys. Lett. 86, $123116(2005)$

[27] S. B. Cronin, Y. Yin, A. Walsh, R. B. Capaz, A. Stolyarov, P. Tangney, M. L. Cohen, S. G. Louie, A.K. Swan, M.S. Ünlü, B.B. Goldberg and M. Tinkham, Phys. Rev. Lett. 96, 127403 (2006)

[28] M. Huang, Y. Wu, B. Chandra, H. Yan, Y. Shan, T. F. Heinz and J. Hone, Phys. Rev. Lett. 100, 136803 (2008)

[29] J.R. Wood, M. D. Frogley, E. R. Meurs, A. D. Prins, T. Peijs, D. J. Dunstan and H. D. Wagner, J. Phys. Chem. B 103, 10388 (1999)

[30] D. Karaiskaj, C. Engtrakul, T. McDonald, M.J. Heben and A. Mascarenhas, Phys. Rev. Lett. 96, 106805 (2006)

[31] K. Arnold, S. Lebedkin, O. Kiowski, F. Hennrich and M.M. Kappes, Nano Lett. 4, 2349 (2004)

[32] Y.K. Kwon, S. Berber and D. Tomanek, Phys. Rev. Lett. 92, 015901 (2004)

[33] H. Tanaka, J. Mol. Liq. 90, 323 (2001)

[34] L. Shen and J. Li, Phys. Rev. B 69,045414 (2004)

[35] T. K. Leeuw, D. A. Tsyboulsky, P. N. Nikolaev, S. M. Bachilo, S. Arepalli and R. B. Weisman, Nanolett. 8, 826 (2008)

[36] R. Saito, G. Dresselhauss, M. S. Dresselhaus, Physical Properties of Carbon Nanotubes, Imperial College Press, 1998

[37] S. Reich, C. Thomsen and J. Maultzsch, Carbon Nanotubes, Wiley-VCH, 2004 